\title{
Clinical characteristics and treatment outcomes of patients with macrolide- resistant Mycobacterium avium complex pulmonary disease: a systematic review and meta-analysis
}

\author{
Youngmok Park', Eun Hye Lee ${ }^{1}$, Inkyung Jung ${ }^{2}$, Goeun Park ${ }^{2}$ and Young Ae Kang ${ }^{1 *}$ (D)
}

\begin{abstract}
Background: Macrolide is a key drug in the treatment of Mycobacterium avium complex pulmonary disease (MACPD). Macrolide-resistant MAC is gaining importance, but there are little data in clinical characteristics and treatment outcomes of macrolide-resistant MAC-PD (MR-MAC-PD).

Methods: We performed a systematic review and meta-analysis of published studies reporting clinical characteristics and treatment outcomes of patients with MR-MAC-PD. Risk of bias was assessed using the modified Newcastle-Ottawa Scale.

Results: Nine studies (seven retrospective and two prospective) comprising 319 patients were identified through a database search. Around $73 \%$ were women, and $52 \%$ had the fibrocavitary form. Pooled sputum culture conversion rate after combined multiple antibiotics or surgical resection was 21\% (95\% confidence interval [Cl], 14-30\%), and the one-year all-cause mortality was 10\% (95\% Cl, 5-20\%). There was no significant difference in treatment outcomes between nodular bronchiectatic and fibrocavitary types.
\end{abstract}

Conclusions: Even combination therapy with fluoroquinolone, aminoglycoside, and surgical resection, the treatment outcomes of MR-MAC-PD were poor. The investigation of new treatment modalities is urgent.

Keywords: Mycobacterium avium complex, Mycobacterium avium-intracellulare infection, Macrolides, Drug resistance, Clarithromycin

\section{Background}

The incidence and prevalence of nontuberculous mycobacterial (NTM) pulmonary disease are increasing worldwide [1-3]. Mycobacterium avium complex (MAC), mainly comprising M. avium and M. intracellulare, has been reported as the most common etiology of NTM pulmonary disease in many countries such as North America and East Asia [3].

\footnotetext{
* Correspondence: mdkang@yuhs.ac

'Division of Pulmonology, Department of Internal Medicine, Severance

Hospital, Yonsei University College of Medicine, 50-1 Yonsei-ro,

Seodaemun-Gu, 03722 Seoul, Republic of Korea

Full list of author information is available at the end of the article
}

Macrolides, such as clarithromycin and azithromycin, are indispensable to the antibiotic treatment of MAC pulmonary disease (MAC-PD). A macrolide-based multidrug regimen comprising ethambutol and rifamycin has been recommended as the first-line therapy for patients with MAC-PD [1, 2, 4]. However, the development of macrolide resistance indicated poor treatment outcomes and increased mortality [5-8], similar to the prognosis of multidrug-resistant tuberculosis (MDR-TB) [7].

Only a few studies with a limited number of patients have evaluated the clinical characteristics and treatment outcomes of macrolide-resistant MAC-PD (MR-MAC$\mathrm{PD})$, and the results were inconsistent in terms of risk factors and optimal treatment modalities. The different 
definitions of treatment outcomes in MAC-PD also led to inconsistency in treatment success. Conducting a prospective controlled study with a large number of patients is challenging because the development of macrolide resistance is unpredictable, and there is no evidence-based treatment regimen in MR-MAC-PD. Therefore, in the present study, we aimed to understand the clinical characteristics and treatment outcomes of MR-MAC-PD through a systematic review and meta-analysis.

\section{Methods}

This study was performed according to the Preferred Reporting Items for Systematic Reviews and MetaAnalyses guidelines [9]. The protocol was registered on PROSPERO (registration number: CRD42019118499).

\section{Search strategies}

We searched Medline, Embase, Cochrane library, and ProQuest databases to identify full-length articles published up to August 25, 2019. The search strategy for each database is presented in Additional file 2: Table S1, S2, S3, and S4. Duplicates, as well as case reports, reviews, conference abstracts, newspaper articles, nonclinical studies, and animal studies were excluded. English studies were selected.

\section{Eligibility criteria}

The inclusion criterion was original studies on MRMAC-PD patients without human immunodeficiency virus (HIV) infection. Relevant studies were independently selected by two reviewers (Y Park and EH Lee). The authors initially screened the articles by title and abstract, and then assessed the full text as needed. Studies with less than 5 patients were excluded. No restrictions were applied regarding study design or methods of data collection (prospective or retrospective).

\section{Data extraction and quality assessment}

Two coding authors (Y Park and EH Lee) extracted data from the selected publications with a pre-defined data extraction form. The following information was recorded: study characteristics (authors, setting, study design, criteria for macrolide resistance), patient characteristics (age, sex, radiologic types, etc.), and treatment outcomes (sputum culture conversion and the one-year all-cause mortality rate). Study quality was assessed using a modified Newcastle-Ottawa Scale [10]. The one-year all-cause mortality rate provided with Kaplan-Meier curve was obtained by digitizing the figure using the online software Web Plot Digitizer [11].

\section{Statistical analysis}

Pooled estimates and 95\% CI were calculated using either fixed-effects or random-effects model.
Heterogeneity was quantified in terms of Q- and $\mathrm{I}^{2}$-statistics. If a significant heterogeneity was present $(P$ values for Q-statistics < 0.10) [12], pooled estimates from random-effects models were reported. Publication bias was assessed using a funnel plot. We used R (v. 3.6.0) in all statistical analyses.

\section{Results}

Study selection and identified studies

A total of 4221 studies were identified from the database search. Among them, 3420 publications were selected after comparison of results and de-duplication. The selected studies were screened by title, abstract, and full text. Figure 1 shows the selection process and exclusion criteria. Finally, we included nine studies on MR-MACPD in this meta-analysis [5-8, 13-17].

The identified studies and their key characteristics are listed in Table 1. There were two prospective studies, and the other seven studies were of retrospective descriptive design. A total of 319 patients were eligible for the analysis. In eight studies, MAC isolates with a clarithromycin minimal inhibitory concentration $\geq 32 \mu \mathrm{g} / \mathrm{mL}$ in the broth microdilution method were defined as macrolide-resistant.

\section{Clinical characteristics}

The mean age of the patients ranged from 65 to 68 years, and the proportion of women ranged from 32 to $100 \%$ (Table 1). The pooled estimate for the proportion of women was $73 \%$ (95\% Confidence interval [CI], 53$86 \%$, Fig. 2a) with random-effects model; $\mathrm{I}^{2}$ statistic was $84 \%$, and Q-statistic was $31.2(P<0.001)$, indicating a high level of heterogeneity.

Five studies reported the percentage of fibrocavitary (FC) type of the disease. The pooled estimate was $52 \%$ (95\% CI, 37-67\%, Fig. 2B) with random-effects model; the $\mathrm{I}^{2}$ statistic was $76 \%$, and Q-statistic was 21.4 $(\mathrm{P}<$ 0.001 ), representing substantial heterogeneity. Four studies $[5-7,14]$ reported the proportion of $M$. avium and $M$. intracellulare separately, and the percentage of $M$. avium ranged from 23 to $100 \%$ (Table 1 ).

As we excluded the studies with HIV infected subjects, most patients had chronic lung diseases such as asthma, chronic obstructive pulmonary disease, bronchiectasis, chronic pulmonary aspergillosis, or history of pulmonary tuberculosis.

\section{Sputum culture conversion rate and the one-year all- cause mortality rate}

The definition of sputum culture conversion in each study is described in Table 2. The reported sputum culture conversion rate ranged from $11 \%[7,16]$ to $38 \%$ [15], and the overall pooled estimate with randomeffects model was $21 \%$ (95\% CI, 14-30\%, Fig. 3a). The $\mathrm{I}^{2}$ 


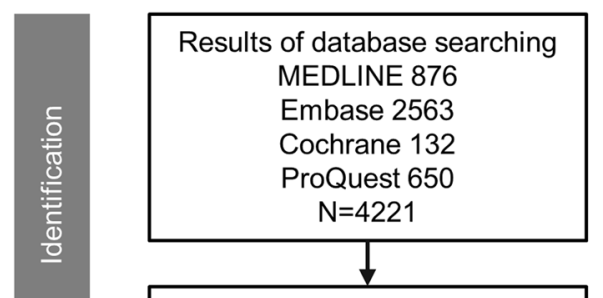

Records available for screening

after duplicates were removed $\mathrm{N}=3420$
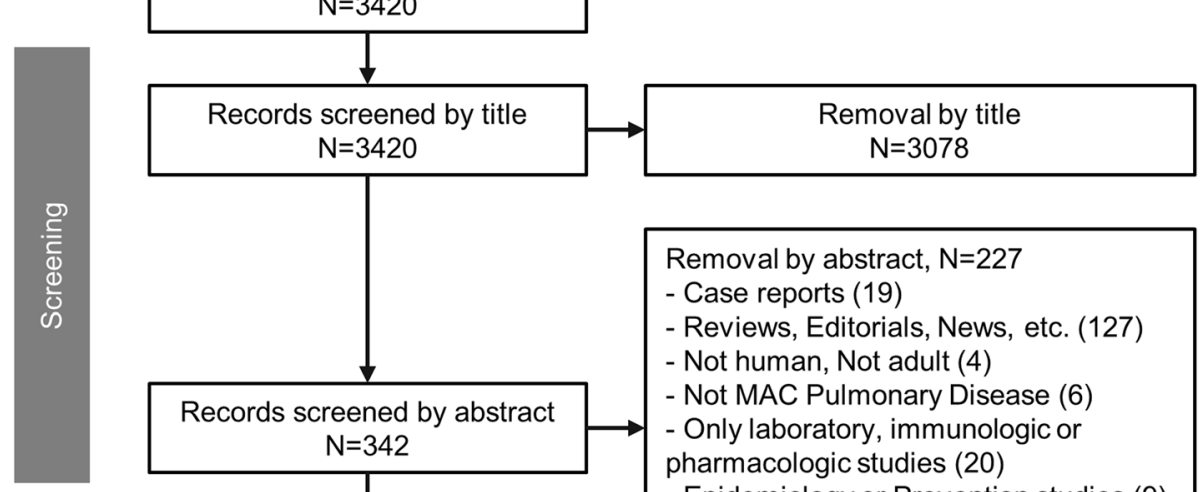

Removal by abstract, $\mathrm{N}=227$

- Case reports (19)

- Reviews, Editorials, News, etc. (127)

- Not human, Not adult (4)

- Not MAC Pulmonary Disease (6)

Records screened by abstract $\mathrm{N}=342$

- Only laboratory, immunologic or

pharmacologic studies (20)

- Epidemiology or Prevention studies (9)

- HIV positive patients (9)

- Not English (25)

- Others (8)
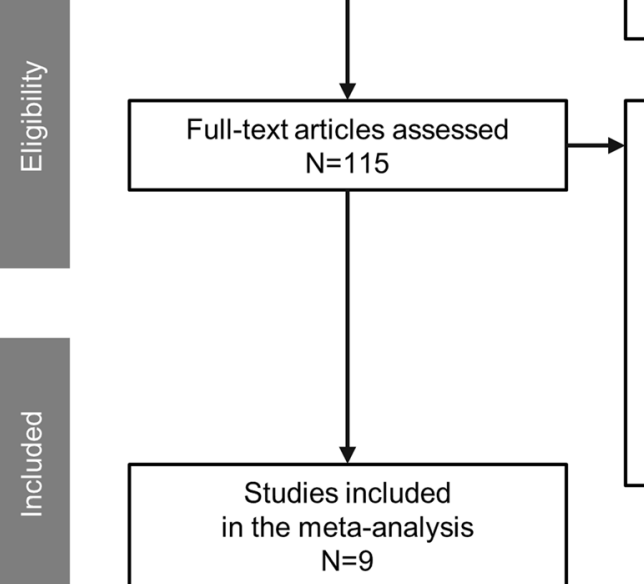

Removal by full-text, $\mathrm{N}=106$

- Incomplete measure of outcomes (10)

- Population less than 5 patients (10)

- Overlap of population (1)

- Treatment for macrolide susceptible patients or no susceptibility data (77)

- Treatment for refractory patients (1)

- Outcome of surgical treatment (5)

- Full-text not available (1)

- Not English (1)

Fig. 1 Flowchart describing the selection of studies included in the analysis. Abbreviations: MAC, M. avium complex pulmonary disease; HIV, human immunodeficiency virus

statistic was $57 \%$, and the Q-statistic was $20.3(P=$ 0.009).

The one-year all-cause mortality rate was reported in four studies (Fig. 3b). It ranged from 0 to 25\% [5], and the pooled estimate was $10 \%(95 \% \mathrm{CI}, 5-20 \%)$ with $\mathrm{I}^{2}$ statistic of $53 \%$ and Q-statistic of $9.60(P=0.048)$.

We conducted a subgroup analysis by radiologic types of MAC-PD. Four studies reported the sputum culture conversion rate between nodular bronchiectatic (NB) and FC types. The estimated odds ratio for sputum culture conversion in NB type compared with the FC type was 0.56 (95\% CI, 0.24-1.30, Additional file 1: Fig. S1A).
Three studies reported the odds ratios for the one-year all-cause mortality rate in NB to FC type: 2.20 (95\% CI, 0.61-7.99) [5]; 0.15 (95\% CI, 0.01-3.19) [6]; and 0.06 (95\% CI, 0.00-1.23) [7]. The pooled estimate was 0.38 (95\% CI, 0.03-4.30) with random-effects model (Additional file 1: Fig. S1B); the interpretation needs caution owing to the limited number of studies.

\section{Treatment modalities of MR-MAC-PD}

According to the studies of Tanaka et al. [13] and Griffith et al. [5], more than half of the patients with MRMAC-PD were treated with macrolide monotherapy or a 


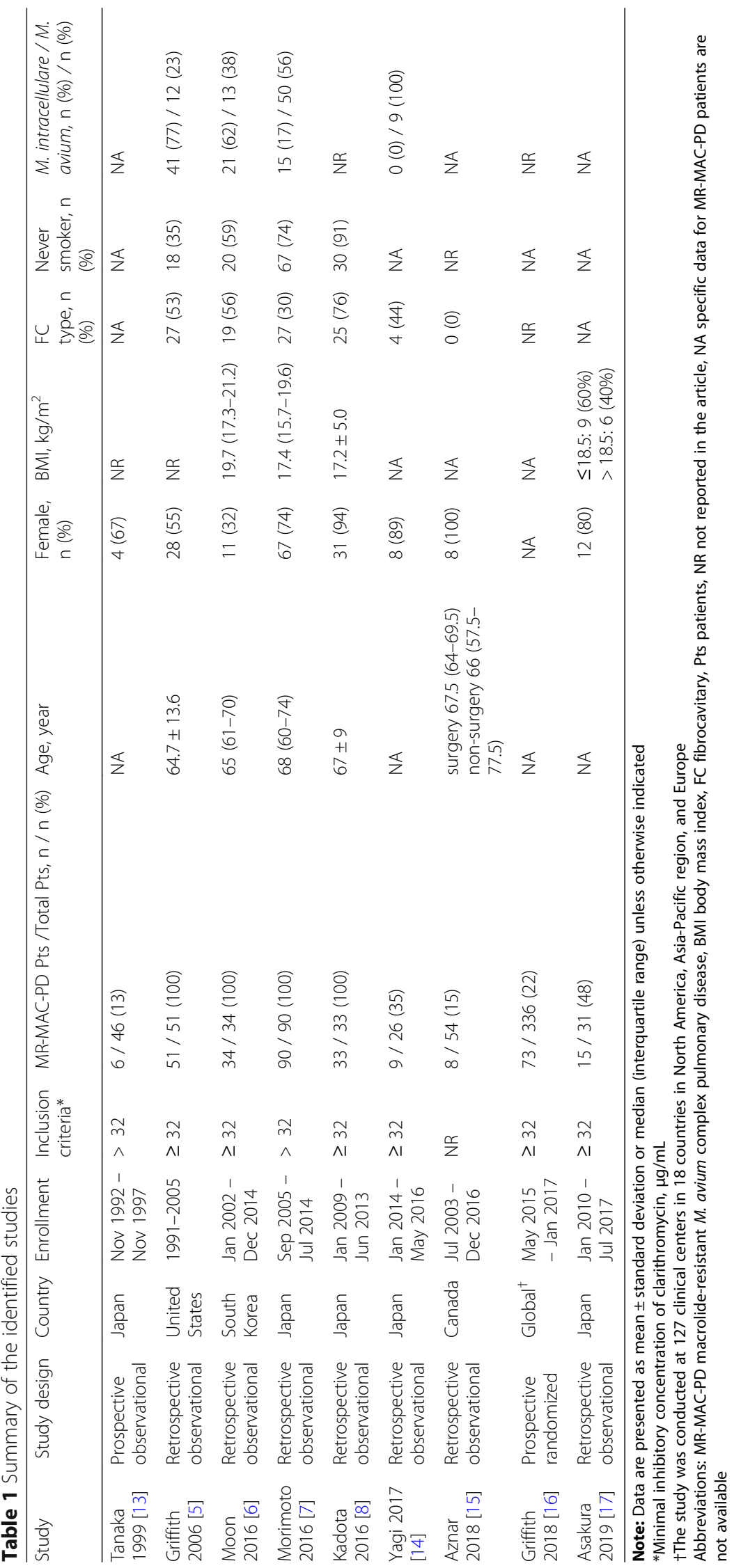




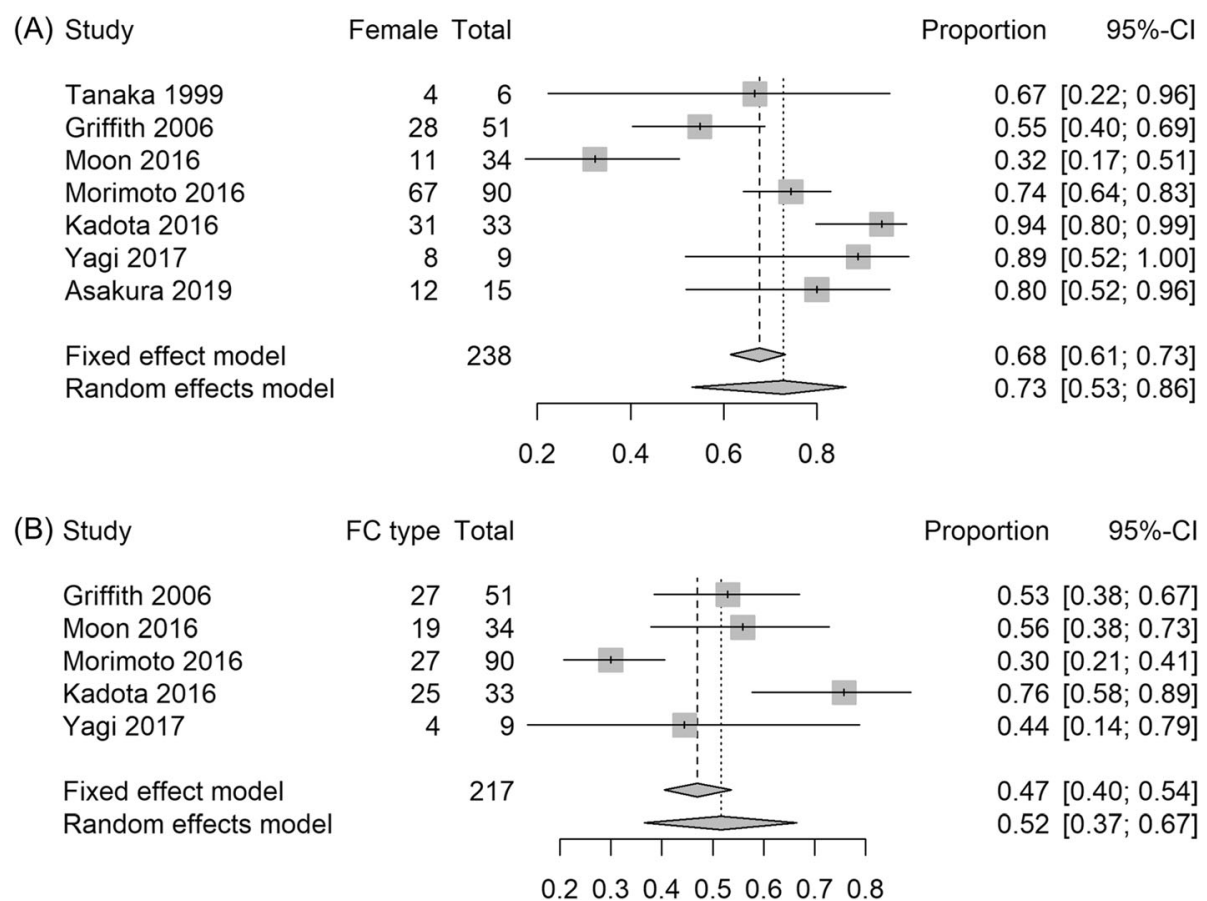

Fig. 2 Clinical characteristics of patients with macrolide-resistant M. avium complex pulmonary disease. Forest plots for (a) female proportion and (b) fibrocavitary (FC) type disease proportion. Note: A study by Aznar et al. [15] was excluded from this analysis because it is designed to match sex and radiologic types

two-drug regimen comprising macrolides before the detection of macrolide resistance. In contrast, patients treated with standard multidrug regimen before the development of macrolide resistance were also substantial in the studies of Moon et al. (65\%) and Morimoto et al. $(28 \%)[6,7]$.

Treatment regimens after the detection of macrolide resistance in each study are presented in Table 2. For the treatment of MR-MAC-PD, new treatment perspectives are tried, such as clofazimine, amikacin inhalation, amikacin liposomal inhalation suspension (ALIS), and surgery.

\section{Assessment of methodological quality}

When methodological quality was assessed using the modified Newcastle-Ottawa Scale, most studies had a low risk of bias for measurement of macrolide resistance, and low to moderate risk of bias in the patient selection and assessment of treatment outcomes (Additional file 2: Table S5).

\section{Discussion}

Newer macrolides, including clarithromycin and azithromycin, are cornerstones in the antibiotic treatment of MAC-PD, and the development of macrolide resistance is associated with poor treatment outcomes [4]. However, there are limited studies on treatment modalities and outcomes for MR-MAC-PD. In this study, we aimed to understand the clinical characteristics and treatment outcomes of MR-MAC-PD through a systematic review and meta-analysis, and found that the overall sputum culture conversion rate was $21 \%$ (95\% CI, 14-30\%), and the one-year all-cause mortality rate was $10 \%(95 \% \mathrm{CI}$, 5-20\%). There were no differences in sputum culture conversion rate and the one-year all-cause mortality rate between NB and FC type of MR-MAC-PD.

The treatment of MAC-PD is complicated, and the treatment results are not satisfactory. Even in macrolidesusceptible MAC-PD, the treatment success rate with macrolide containing multidrug regimens ranged from $60 \%$ [18] to $65.7 \%$ [19]. Sustained sputum culture conversion rate with macrolide-free regimens was $38 \%$ in a previous systematic review [20]. In our present study, the pooled estimate of sputum culture conversion rate was $21 \%$ (95\% CI, 14-30\%), which represents a worse treatment outcome of MR-MAC-PD. Pan et al. reported that microbiologic persistence in patients with MAC-PD could lead to an increased risk of radiographic progression [21]. In the same perspective, low sputum culture conversion rate in MR-MAC-PD could be a predictor of disease progression including radiographic progression.

Patients with MAC-PD are at a significant risk of death. Diel et al. [22] reported a pooled estimate of the five-year all-cause mortality rate of patients with MACPD as 27\% (95\% CI, 21-33\%). Moon et al. [6] and Morimoto et al. [7] reported five-year all-cause mortality 


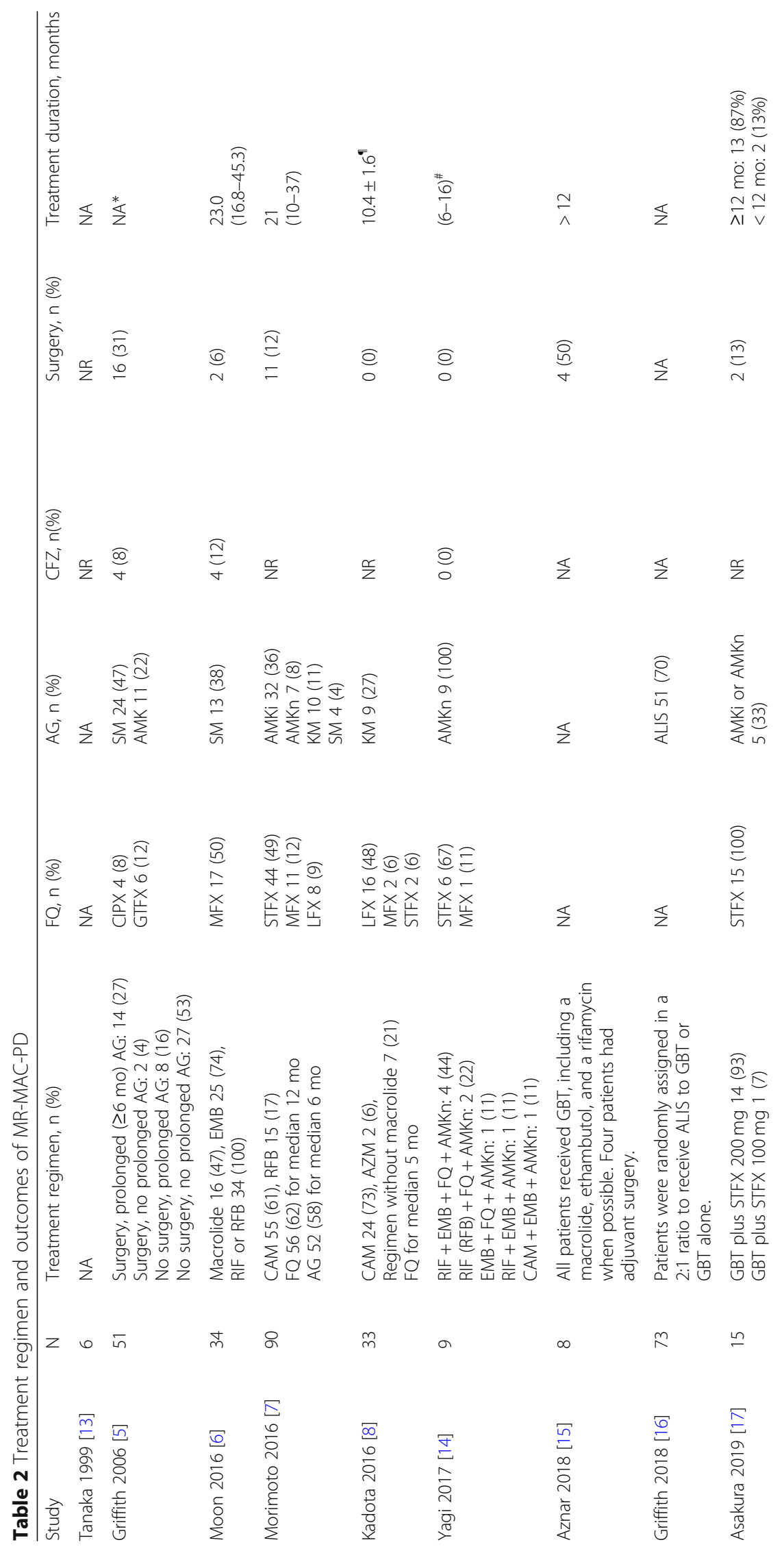




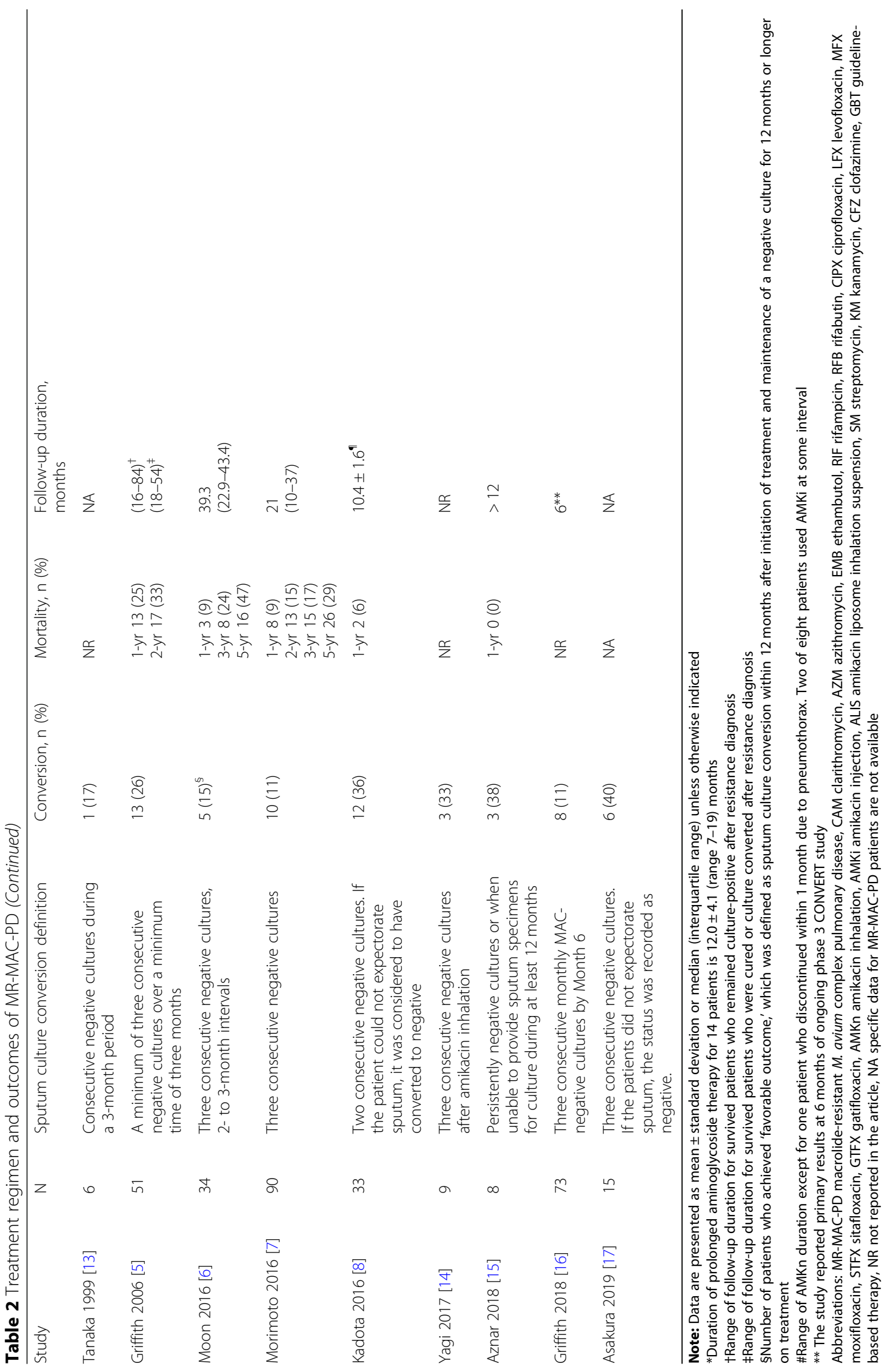




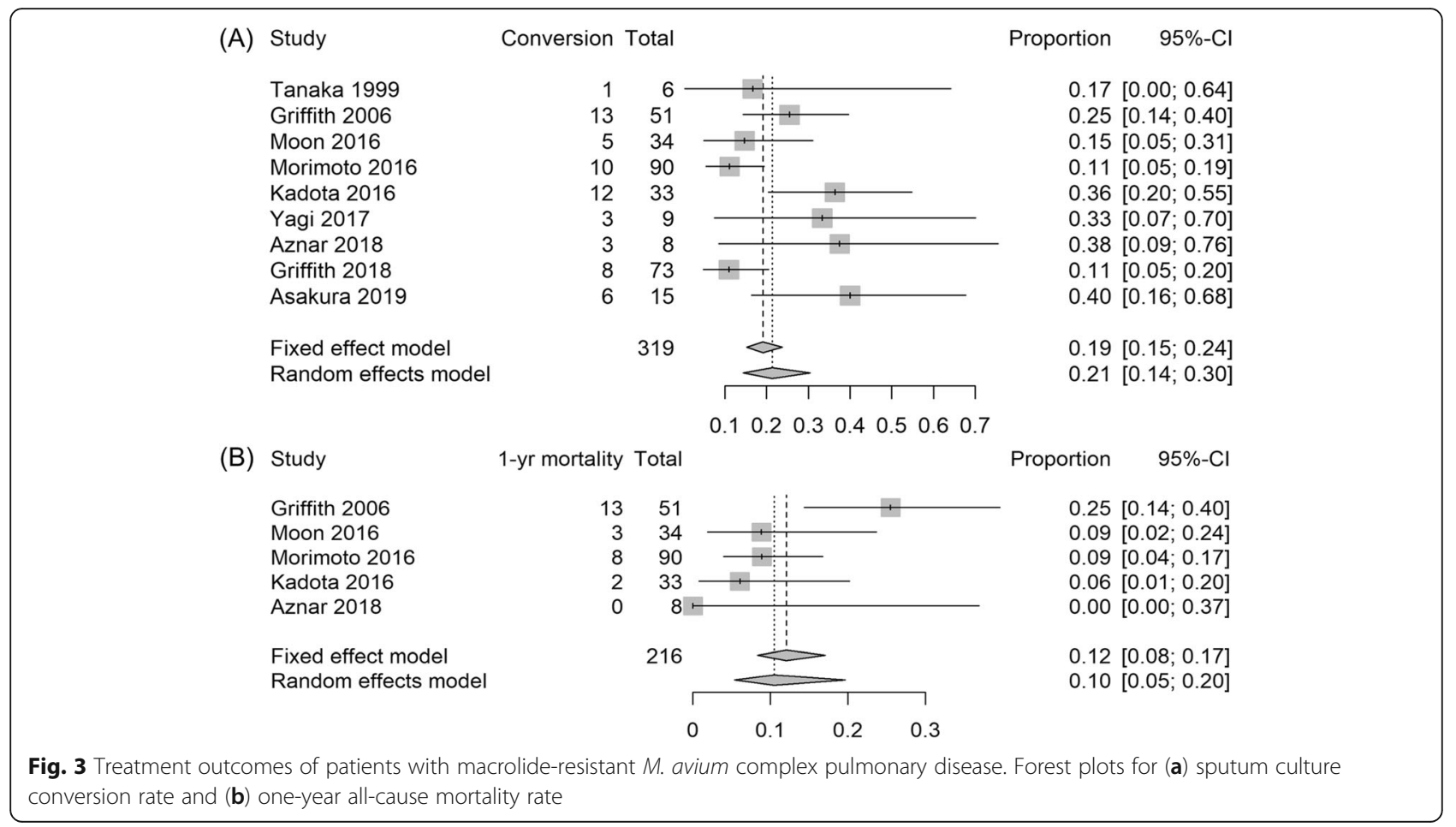

rates of MR-MAC-PD as 47 and 29\%, respectively. The pooled estimate of the one-year mortality rate was $10 \%$ (95\% CI, 5-20\%) in this study. Several studies have demonstrated the incremental impact of NTM infection on mortality [23-25]; however, the assessment of NTMrelated mortality is difficult because the proportion of deaths attributed to NTM infection depends mostly on how clinicians decide the cause of death. In addition, there are limited data on the mortality of MR-MAC-PD compared to the general population and patients with macrolide-susceptible MAC-PD. Considering the chronic features of NTM infection, long-term mortality assessment is necessary for patients with MR-MAC-PD.

Morimoto et al. [7] compared the treatment outcome of MR-MAC-PD to that of 311 patients with MDR-TB; the 5-year survival rates between the two groups were similar (71\% vs. $75 \%, P=0.6$ ). MDR-TB is considered a severe health concern worldwide. The World Health Organization reported that only $55 \%$ of patients with MDR-TB in 2015 successfully completed medication; the treatment failed in $8 \%$ of the patients, and $15 \%$ died [26].

There are a few explanations for the emergence of macrolide resistance in MAC-PD. First, inappropriate regimen as first-line treatment may trigger macrolide resistance. Griffith et al. [5] reported that the majority (76\%) of patients with MR-MAC-PD started their initial treatment with macrolide monotherapy or the combination of a macrolide and a fluoroquinolone. Morimoto et al. [7] showed that $60.2 \%$ of patients did not receive proper multidrug regimens, such as clarithromycin monotherapy, clarithromycin plus fluoroquinolone, and regimens without ethambutol. Moon et al. [6] also reported that one-third of the population did not receive ethambutol owing to its adverse events.

Second, relatively low concentrations of core drugs and high bacterial burden have been suggested as an explanation for macrolide resistance. Concomitant use of rifamycin is related to reduced serum levels of macrolide, particularly clarithromycin [27, 28]. Moon et al. [6] and Kadota et al. [8] reported that macrolide resistance can occur even when patients were treated with proper multidrug regimen, because a small proportion of patients received macrolide monotherapy (32\% in the study of Moon et al. and 18\% in the study of Kadota et al., respectively) or a two-drug combination with a macrolide (33\% in the study of Moon et al. and $12 \%$ in the study of Kadota et al., respectively).

There is no proven treatment modality for MR-MACPD yet. Maintenance of macrolide after the detection of macrolide resistance is frequent. Surgical intervention and prolonged parenteral aminoglycoside administration are the primary treatment strategies for the treatment of MR-MAC-PD (Table 2). The recent British Thoracic Society guidelines recommend adding another drug such as isoniazid, moxifloxacin, or nebulized amikacin for treatment of MR-MAC-PD. However, the efficacy of these treatment regimens remains inconclusive [2]. Recently, clofazimine, bedaquiline, and ALIS have been used for the treatment of refractory MAC-PD, including MR-MAC-PD [16, 29, 30]. In the CONVERT study [16], 
a prospective open-label, randomized study for the treatment of patients with refractory MAC-PD, ALIS with guideline-based therapy (GBT) showed culture conversion rate of $29.0 \%$ compared to $8.9 \%$ of GBT alone. Among MR-MAC-PD group, culture conversion was achieved by $13.7 \%$ of patients in the ALIS + GBT arm and $4.5 \%$ in the GBT-alone arm. Even this latest ALIS therapy, treatment outcome of MR-MAC-PD was poor. Bedaquiline was also recently tried as a treatment option for refractory NTM-PD, although the study was preliminary and the number of patients was small [29]. Therefore, for the treatment of MR-MAC-PD, new drugs or new pharmaceutical formulations of existing drugs should be investigated.

This is the first study to integrate the clinical characteristics and treatment outcomes of patients with MRMAC-PD. Nevertheless, this study has several limitations. First, because only a small number of studies were enrolled in this analysis, we could not thoroughly evaluate the publication bias. Second, all the studies had no control group, and most of them were retrospective observational design. Therefore, we should interpret the results with caution. Third, the treatment outcomes were estimated from the various treatment modalities because there was no uniform protocol for the treatment of MRMAC-PD. Fourth, we could not evaluate the long-term treatment outcomes of MR-MAC-PD owing to the relatively short follow-up duration of each study. In addition, we could not assess treatment outcomes including clinical and radiographic improvement.

In conclusion, the treatment outcomes of MR-MACPD were poor, the overall sputum culture conversion rate was $21 \%(95 \% \mathrm{CI}, 14-30 \%)$, and the one-year allcause mortality rate was 10\% (95\% CI, 5-20\%). Despite the combination of multiple antibiotics including ALIS and surgical resection, the outcomes of MR-MAC-PD were poor The investigation of new treatment modalities is urgent for the treatment of MR-MAC-PD.

\section{Supplementary information}

Supplementary information accompanies this paper at https://doi.org/10. 1186/s12931-019-1258-9.

Additional file 1: Fig. S1 Comparison of (A) sputum culture conversion rate and (B) one-year all-cause mortality rate between nodular bronchiectatic (NB) and fibrocavitary (FC) type disease of macrolide-resistant $M$.

avium complex pulmonary disease

Additional file 2: Table S1. Database search strategy for MEDLINE. Table S2 Database search strategy for Embase. Table S3 Database search strategy for Cochrane library. Table \$4 Database search strategy for ProQuest. Table S5 Quality assessment of included studies using a modified Newcastle-Ottawa scale

\section{Abbreviations}

ALIS: Amikacin liposomal inhalation suspension; Cl: Confidence interval; FC: Fibrocavitary; GBT: Guideline-based therapy; HIV: Human immunodeficiency virus; MAC: Mycobacterium avium complex; MAC-
PD: Mycobacterium avium complex pulmonary disease; MDR-TB: Multidrugresistant tuberculosis; MR-MAC-PD: Macrolide-resistant Mycobacterium avium complex pulmonary disease; NB: Nodular bronchiectatic;

NTM: Nontuberculous mycobacteria

\section{Acknowledgments}

Not applicable.

\section{Authors' contributions}

YP database search, data extraction, manuscript writing. EHL database search, data extraction. IJ statistical analysis. GP statistical analysis. YAK overall management. All authors read and approved the final manuscript.

\section{Funding}

This research did not receive any specific grant from funding agencies in the public, commercial, or not-for-profit sectors.

\section{Availability of data and materials}

This study was a re-analysis of existing data, which is openly available at locations cited in the reference section.

Ethics approval and consent to participate

Not applicable.

\section{Consent for publication}

Not applicable.

\section{Competing interests}

The authors declare that they have no competing interests.

\section{Author details}

${ }^{1}$ Division of Pulmonology, Department of Internal Medicine, Severance Hospital, Yonsei University College of Medicine, 50-1 Yonsei-ro, Seodaemun-Gu, 03722 Seoul, Republic of Korea. ${ }^{2}$ Division of Biostatistics, Department of Biomedical Systems Informatics, Yonsei University College of Medicine, Seoul, Republic of Korea.

Received: 26 April 2019 Accepted: 9 December 2019

Published online: 18 December 2019

\section{References}

1. Griffith DE, Aksamit T, Brown-Elliott BA, Catanzaro A, Daley C, Gordin F, Holland SM, Horsburgh R, Huitt G, lademarco MF, et al. An official ATS/IDSA statement: diagnosis, treatment, and prevention of nontuberculous mycobacterial diseases. Am J Respir Crit Care Med. 2007;175:367-416.

2. Haworth CS, Banks J, Capstick T, Fisher AJ, Gorsuch T, Laurenson IF, Leitch A Loebinger MR, Milburn HJ, Nightingale M, et al. British Thoracic Society guideline for the management of non-tuberculous mycobacterial pulmonary disease (NTM-PD). BMJ Open Respir Res. 2017;4:e000242.

3. Prevots DR, Marras TK. Epidemiology of human pulmonary infection with nontuberculous mycobacteria: a review. Clin Chest Med. 2015;36:13-34.

4. Kwon YS, Koh WJ, Daley CL. Treatment of Mycobacterium avium complex pulmonary disease. Tuberc Respir Dis Seoul. 2019;82:15-26.

5. Griffith DE, Brown-Elliott BA, Langsjoen B, Zhang Y, Pan X, Girard W, Nelson K, Caccitolo J, Alvarez J, Shepherd S, et al. Clinical and molecular analysis of macrolide resistance in Mycobacterium avium complex lung disease. Am J Respir Crit Care Med. 2006;174:928-34.

6. Moon SM, Park HY, Kim SY, Jhun BW, Lee H, Jeon K, Kim DH, Huh HJ, Ki CS, Lee NY, et al. Clinical characteristics, treatment outcomes, and resistance mutations associated with macrolide-resistant Mycobacterium avium complex lung disease. Antimicrob Agents Chemother. 2016;60:6758-65.

7. Morimoto K, Namkoong H, Hasegawa N, Nakagawa T, Morino E, Shiraishi $Y$, Ogawa K, Izumi K, Takasaki J, Yoshiyama T, et al. Macrolide-resistant Mycobacterium avium complex lung disease: analysis of 102 consecutive cases. Ann Am Thorac Soc. 2016;13:1904-11

8. Kadota T, Matsui H, Hirose T, Suzuki J, Saito M, Akaba T, Kobayashi K, Akashi S, Kawashima M, Tamura A, et al. Analysis of drug treatment outcome in clarithromycin-resistant Mycobacterium avium complex lung disease. BMC Infect Dis. 2016;16:31. 
9. Moher D, Liberati A, Tetzlaff J, Altman DG. Preferred reporting items for systematic reviews and meta-analyses: the PRISMA statement. Open Med. 2009;3:e123-30.

10. The Newcastle-Ottawa Scale (NOS) for assessing the quality of nonrandomised studies in meta-analyses [http://www.ohri.ca/programs/ clinical_epidemiology/oxford.asp].

11. Rohatgi A: WebPlotDigitizer, v4.1. 2018.

12. Hedges L, Olkin, I.: Statistical methods for Meta-Analysis. 1st edn: Academic Press; 2014.

13. Tanaka E, Kimoto T, Tsuyuguchi K, Watanabe I, Matsumoto H, Niimi A, Suzuki K, Murayama T, Amitani R, Kuze F. Effect of clarithromycin regimen for Mycobacterium avium complex pulmonary disease. Am J Respir Crit Care Med. 1999;160:866-72.

14. Yagi $K$, Ishii M, Namkoong H, Asami T, Iketani O, Asakura T, Suzuki S, Sugiura $H$, Yamada $Y$, Nishimura $T$, et al. The efficacy, safety, and feasibility of inhaled amikacin for the treatment of difficult-to-treat non-tuberculous mycobacterial lung diseases. BMC Infect Dis. 2017;17:558.

15. Aznar ML, Zubrinic M, Siemienowicz M, Hashimoto K, Brode SK, Mehrabi M, Patsios D, Keshavjee S, Marras TK. Adjuvant lung resection in the management of nontuberculous mycobacterial lung infection: a retrospective matched cohort study. Respir Med. 2018;142:1-6.

16. Griffith DE, Eagle G, Thomson R, Aksamit TR, Hasegawa N, Morimoto K, Addrizzo-Harris DJ, O'Donnell AE, Marras TK, Flume PA, et al. Amikacin liposome inhalation suspension for treatment-refractory lung disease caused by Mycobacterium avium complex CONVERT. A prospective, openlabel, randomized study. Am J Respir Crit Care Med. 2018;198:1559-69.

17. Asakura T, Suzuki S, Fukano H, Okamori S, Kusumoto T, Uwamino Y, Ogawa T, So M, Uno S, Namkoong $H$, et al. Sitafloxacin-containing regimen for the treatment of refractory mycobacterium avium complex lung disease. Open Forum Infectious Diseases. 2019;6.

18. Kwak N, Park J, Kim E, Lee CH, Han SK, Yim JJ. Treatment outcomes of Mycobacterium avium complex lung disease: a systematic review and meta-analysis. Clin Infect Dis. 2017;65:1077-84.

19. Diel R, Nienhaus A, Ringshausen FC, Richter E, Welte T, Rabe KF, Loddenkemper R. Microbiologic outcome of interventions against Mycobacterium avium complex pulmonary disease: a systematic review. Chest. 2018;153:888-921.

20. Pasipanodya JG, Ogbonna D, Deshpande D, Srivastava S, Gumbo T. Metaanalyses and the evidence base for microbial outcomes in the treatment of pulmonary Mycobacterium avium-intracellulare complex disease. J Antimicrob Chemother. 2017;72:i3-i19.

21. Pan SW, Shu CC, Feng JY, Wang JY, Chan YJ, Yu CJ, Su WJ. Microbiological persistence in patients with Mycobacterium avium complex lung disease: the predictors and the impact on radiographic progression. Clin Infect Dis. 2017;65:927-34.

22. Diel R, Lipman M, Hoefsloot W. High mortality in patients with Mycobacterium avium complex lung disease: a systematic review. BMC Infect Dis. 2018;18:206

23. Andrejak C, Thomsen VO, Johansen IS, Riis A, Benfield TL, Duhaut $P$, Sorensen HT, Lescure FX, Thomsen RW. Nontuberculous pulmonary mycobacteriosis in Denmark: incidence and prognostic factors. Am J Respir Crit Care Med. 2010;181:514-21.

24. Marras TK, Campitelli MA, Lu H, Chung H, Brode SK, Marchand-Austin A, Winthrop KL, Gershon AS, Kwong JC, Jamieson FB. Pulmonary Nontuberculous mycobacteria-associated deaths, Ontario, Canada, 20012013. Emerg Infect Dis. 2017;23:468-76.

25. Novosad SA, Henkle E, Schafer S, Hedberg K, Ku J, Siegel SAR, Choi D, Slatore CG, Winthrop KL. Mortality after Respiratory Isolation of Nontuberculous Mycobacteria. A comparison of patients who did and did not meet disease criteria. Ann Am Thorac Soc. 2017;14:1112-9.

26. World Health Organization: Global tuberculosis report 2018 (WHO/CDS/TB/ 2018.20). 2018

27. Koh WJ, Jeong BH, Jeon K, Lee SY, Shin SJ. Therapeutic drug monitoring in the treatment of Mycobacterium avium complex lung disease. Am J Respir Crit Care Med. 2012;186:797-802.

28. van Ingen J, Egelund EF, Levin A, Totten SE, Boeree MJ, Mouton JW Aarnoutse RE, Heifets LB, Peloquin CA, Daley CL. The pharmacokinetics and pharmacodynamics of pulmonary Mycobacterium avium complex disease treatment. Am J Respir Crit Care Med. 2012;186:559-65.

29. Philley JV, Wallace RJ Jr, Benwill JL, Taskar V, Brown-Elliott BA, Thakkar F, Aksamit TR, Griffith DE. Preliminary results of Bedaquiline as salvage therapy for patients with Nontuberculous mycobacterial lung disease. Chest. 2015 148:499-506.

30. Martiniano SL, Wagner BD, Levin A, Nick JA, Sagel SD, Daley CL. Safety and effectiveness of Clofazimine for primary and refractory Nontuberculous mycobacterial infection. Chest. 2017;152:800-9.

\section{Publisher's Note}

Springer Nature remains neutral with regard to jurisdictional claims in published maps and institutional affiliations.
Ready to submit your research? Choose BMC and benefit from:

- fast, convenient online submission

- thorough peer review by experienced researchers in your field

- rapid publication on acceptance

- support for research data, including large and complex data types

- gold Open Access which fosters wider collaboration and increased citations

- maximum visibility for your research: over $100 \mathrm{M}$ website views per year

At $\mathrm{BMC}$, research is always in progress.

Learn more biomedcentral.com/submissions 